\title{
Radiative Feedback in Galaxies
}

\author{
M. S. Oey ${ }^{1}$, E. S. Voges $^{2}$, R. A. M. Walterbos ${ }^{2}$, G. R. Meurer ${ }^{3}$ \\ S. Yelda ${ }^{4}$ and E. Furst ${ }^{5}$ \\ ${ }^{1}$ Department of Astronomy, 830 Dennison Building, University of Michigan, Ann Arbor, MI \\ 48109-1042, U.S.A. \\ ${ }^{2}$ Department of Astronomy, MSC 4500, New Mexico State University, P.O. Box 30001, Las \\ Cruces, NM 88003, U.S.A. \\ ${ }^{3}$ Johns Hopkins University, Department of Physics and Astronomy, 3400 North Charles Street, \\ Baltimore, MD 21218-2686, U.S.A. \\ ${ }^{4}$ University of California, Department of Physics and Astronomy, P.O. Box 951547, Los \\ Angeles, CA 90095-1547, U.S.A. \\ ${ }^{5} 344$ Greenlow Road, Catonsville, MD 21228, U.S.A.
}

\begin{abstract}
We examine the fate of ionizing radiation from massive stars on global scales. First, we compare the observed $\mathrm{H} \alpha$ luminosities of LMC HiI regions with those predicted by the latest generation of stellar atmosphere models. Our results imply that classical His regions are on average radiation-bounded, rather than density-bounded, as we found a decade ago. This is likely to necessitate an additional ionizing source for the diffuse, warm ionized medium (WIM) in galaxies. Secondly, we present new results from the SINGG H $\alpha$ galaxy survey, showing that starburst galaxies have a lower fraction of WIM emission than normal star-forming galaxies. The most intriguing and consequential possible cause for this effect is the escape of ionizing radiation from starbursts. We show that the observations are also consistent with our predictions for the escape of ionizing radiation. Nevertheless, other observations do not necessarily support this scenario and other possible explanations must be considered.
\end{abstract}

Keywords. stars: atmospheres - stars: early-type - galaxies: evolution - galaxies: ISM - galaxies: starburst - HII regions - intergalactic medium - Magellanic Clouds

\section{Introduction}

The preceding speakers have described how mechanical and radiative feedback are intrinsically linked. Here, we will focus on the radiative feedback from massive stars, which refers specifically to the resulting photoionization of the gaseous environment. Classical Hir regions are the most obvious manifestation, but another consequence is the photoionization of the interstellar medium (ISM) itself, resulting in the diffuse, warm ionized medium (WIM). In the case of starbursts or extreme star-formation intensity, ionizing radiation could escape from the galaxies altogether, ionizing the galactic halo, IGM, or even the cosmos itself.

\section{Implications of Recent Stellar Atmosphere Models}

Over the past decade, we have understood the ionization of the WIM in terms of a coherent picture: About half of the power is due to photoionization by field OB stars (Hoopes \& Walterbos 2000; Oey et al. 2004), and the other half due to UV radiation escaping from density-bounded HiI regions (Oey \& Kennicutt 1997; Hoopes et al. 2000). Some of the strongest evidence pointing to the role of the HiI regions was our work from ten years ago, in which we compared the observed $\mathrm{H} \alpha$ luminosities of a sample of nebulae 
in the Large Magellanic Cloud (LMC) with the values predicted by model atmospheres for the individual, spectroscopically classified stars observed in these regions (Oey \& Kennicutt 1997). As is well-known in the massive star community, the more recent stellar atmosphere models are softer and predict less Lyman continuum radiation. We therefore decided to revisit the issue of radiative feedback in light of these newer models.

We take the same sample of 14 LMC HII regions from Oey \& Kennicutt (1997) and now use the modern predictions from stellar atmosphere models by Smith, Norris, \& Crowther (2002) and Martins, Schaerer, and Hillier (2005). The Smith et al. values are the most widely-used since they are incorporated into the STARBURST99 population synthesis code. The results are compared to those reported by Oey \& Kennicutt (1997), which were based on ionizing emission rates predicted by Schaerer \& de Koter (1997), Vacca, Garmany, \& Shull (1996), and Panagia (1973).

The newer models predict roughly half the ionizing photon emission rates $Q^{0}$ relative to the older models. Figure 1 compares the observed $\mathrm{H} \alpha$ luminosities with the predicted values for the sample nebulae. Diamonds and squares correspond to predictions based on the Smith et al. (2002) and Martins et al. (2005) models, respectively. Points with crosses represent objects that are partly shock-ionized, and so their $\mathrm{H} \alpha$ luminosities are higher than expected simply from photoionization. Ignoring these shock-ionized objects, Figure 1 shows excellent agreement between the observed and predicted $\mathrm{H} \alpha$ luminosities. The deviation from the identity relation in this logarithmic plot, excluding the shockionized objects, is -0.031 and +0.122 for the Smith et al. (2002) and Martins et al. (2005) predictions, respectively. These correspond to linear observed-to-predicted ratios of 0.92 and 1.3, respectively, for the two sets of predictions. We furthermore examined a set of 39 LMC HII regions with stellar spectral types estimated photometrically. These estimates are less reliable than actual classifications, but the results are consistent with Figure 1 (Voges et al. 2008).

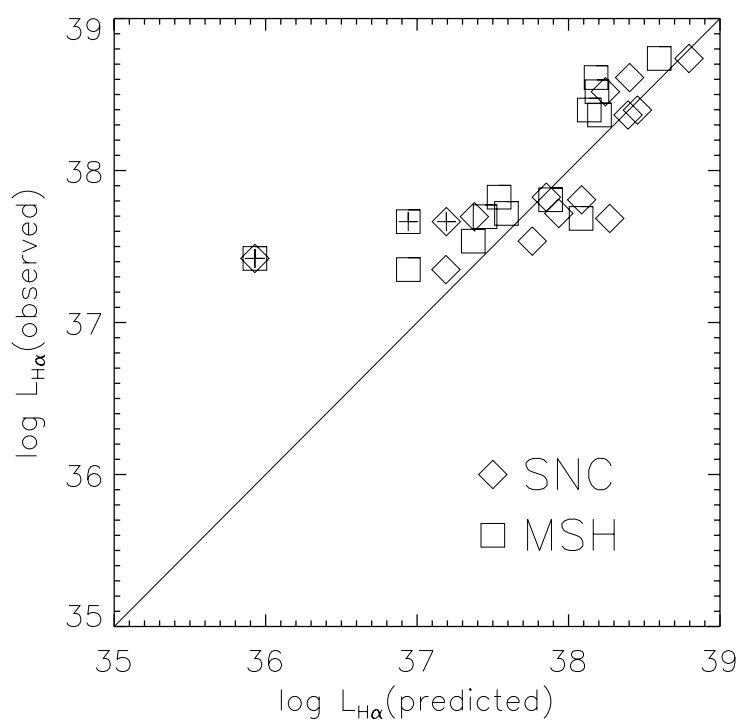

Figure 1. Comparison of observed and predicted $\mathrm{H} \alpha$ luminosities for the LMC HII regions. Diamonds and squares show the comparisons for the Smith et al. (2002) and Martins et al. (2005) predictions, respectively. Objects identified with '+' signs have significant additional contributions from shock ionization. (From Voges et al. 2008.) 
While these newer models solve some outstanding problems for understanding massive stars, this however causes a new problem for understanding the ISM: These results imply that density-bounded HiI regions may not be able to account for the required $50 \%$ ionization of the WIM. Figure 1 does show a large scatter, a factor of $\sim 1.5$, and so many objects are still likely to be density-bounded. However, the bulk reduction in available ionizing radiation by this mechanism requires other processes to help power the WIM.

\section{Ionizing Radiation from Starbursts}

We now turn to radiative feedback from galaxies, an issue of vital importance in understanding the reionization of the universe and galactic energy budgets. The Survey of Ionization in Neutral-Gas Galaxies (SINGG; Meurer et al. 2006) is an $\mathrm{H} \alpha$ and $R$-band survey of HI-selected galaxies aimed at understanding the local star-formation rate density and star-forming properties of galaxies in the local universe. The galaxies are selected to uniformly sample Hi gas masses in the range $10^{7}$ to $10^{11} M_{\odot}$. The results reported here and by Oey et al. (2007) are based on the first data release of 109 galaxies.

We categorized the galaxies according to star-formation intensity (SFI), defined as the $\mathrm{H} \alpha$ surface brightness $\Sigma_{\mathrm{H} \alpha}$ within the $\mathrm{H} \alpha$ half-light radius. Galaxies having $\log \Sigma_{\mathrm{H} \alpha}>$ 39.4 are categorized as "starburst" galaxies, and those having $\log \Sigma_{\mathrm{H} \alpha} \leqslant 38.4$ are denoted as galaxies having "sparse" star formation; galaxies with $\Sigma_{\mathrm{H} \alpha}$ between these boundaries are considered "normal". Furthermore, many galaxies have almost all of their star formation and $\mathrm{H} \alpha$ emission concentrated in the nuclear regions, and so we noted such galaxies in addition. We used the HIIphot code (Thilker et al. 2000) to identify the boundaries of the individual Hı regions and thereby define the allocation of $\mathrm{H} \alpha$ emission between the classical HII regions and the WIM. Note that HIIphot does account for background diffuse emission associated with the identified HiI regions.

Our study (Oey et al. 2007) examines a variety of parameters, but the most notable result is an anti-correlation between the fraction of total $\mathrm{H} \alpha$ luminosity occupied by the WIM and the star-formation intensity, shown in Figure 2. The relation is apparent for starburst galaxies in particular, recalling that these are systems having $\log \Sigma_{\mathrm{H} \alpha}>39.4$. The effect is also seen in the $\mathrm{H} \alpha$ surface brightness distributions: Our starburst galaxies

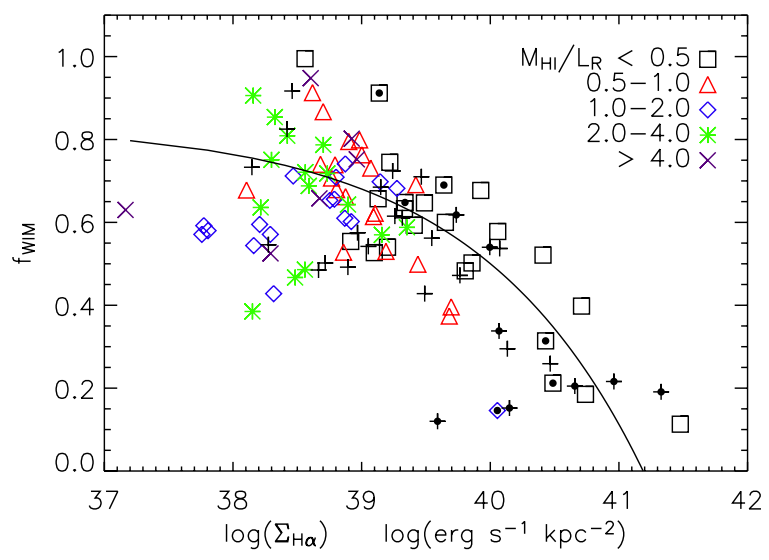

Figure 2. Fraction of diffuse $\mathrm{H} \alpha$ emission as a function of $\mathrm{H} \alpha$ surface brightness, a measure of star-formation intensity. Symbols show Hi mass relative to $R$-band luminosity, a measure of Hi gas fraction, as shown. (From Oey et al. 2007.) 
show much shallower $\mathrm{H} \alpha$ surface brightness distributions than those for the "normal" and "sparse" star-formation categories (see Oey et al. 2007).

What is the origin of this intriguing decrease in WIM fraction with SFI for starbursts? There are two general possibilities: $a$ ) The sources of ionizing radiation are systematically reduced; or $b$ ) The starbursts are fully occupying the ISM, thereby leaving progressively less neutral, diffuse ISM to be ionized. The latter implies that the ISM is essentially density-bounded, allowing excess ionizing radiation to escape from the galaxy disks. It would be an important consequence if this radiation escapes from the galaxies altogether, allowing ionization of the intergalactic environment, and the cosmos itself.

In evaluating these two possibilities, note that starbursts have a morphological appearance that is consistent with this second scenario. The giant star-formation regions are closely packed together, leaving little external ISM that could serve as diffuse WIM (Figure 3). Clarke \& Oey (2002) predicted a threshold critical star-formation rate (SFR) above which the mechanical feedback shreds the ISM, allowing the escape of ionizing radiation and hot, metal-laden superwinds. This is simply a balance of the ISM weight against the force of mechanical feedback. In terms of the global galaxy parameters, our rough estimate of this criterion is:

$$
\mathrm{SFR}_{\text {crit }} \sim 0.15\left(M_{\mathrm{ISM}, 10} \tilde{v}_{10}^{2} / f_{d}\right) \quad \mathrm{M}_{\odot} \mathrm{yr}^{-1},
$$

where $M_{\text {ISM,10 }}$ is the total ISM mass in units of $10^{10} \mathrm{M}_{\odot}, \tilde{v}_{10}$ is the thermal/turbulent velocity dispersion in units of $10 \mathrm{~km} \mathrm{~s}^{-1}$, and $f_{d}$ is a geometric correction factor for disk galaxies of order unity. Applying equation 3.1 to local starbursts, we find that typical critical SFR's are $\lesssim 1 \mathrm{M}_{\odot} \mathrm{yr}^{-1}$, whereas actual SFR's are more like $1-20 \mathrm{M}_{\odot} \mathrm{yr}^{-1}$. This implies that in principle, local starbursts should largely be meeting this criterion, and allowing the escape of outflows and ionizing radiation. Figure 4 shows the $\mathrm{H} \alpha$ luminosities, relative to the value corresponding to the critical SFR, as a function of SFI for our sample galaxies. It is apparent that the galaxies with the highest SFI all exceed the threshold

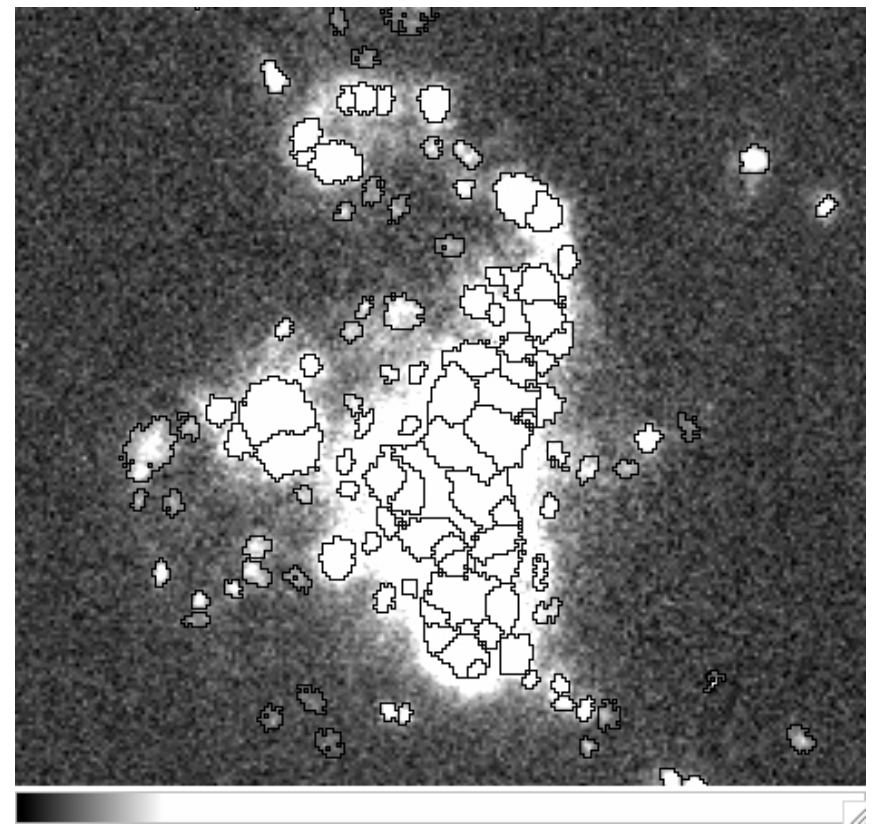

Figure 3. Continuum-subtracted $\mathrm{H} \alpha$ image of starburst galaxy J0355-42, showing HiI region boundaries identified by the HIIphot software. (From Oey et al. 2007) 


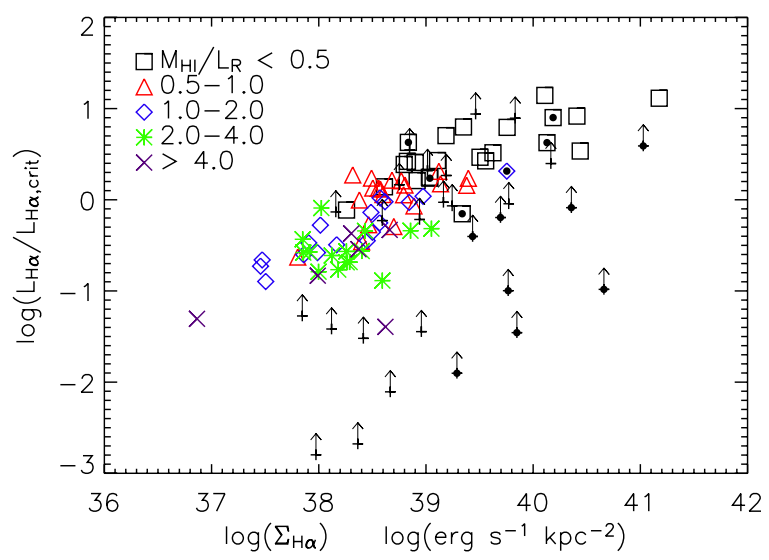

Figure 4. Total $\mathrm{H} \alpha$ luminosity, a measure of star-formation rate, relative to the critical threshold for dominating feedback. Symbols are as in Figure 2. (From Oey et al. 2007.)

criterion. We do caution that equation 3.1 is a crude parameterization, which is likely to need refinement and calibration. However, the general trend is fully consistent with an expectation that starburst galaxies should allow the escape of ionizing radiation. Moreover, both Figures 2 and 4 demonstrate that galaxies with low fractions of diffuse emission are also those that have the lowest Hi gas fractions, which is also consistent with their ISM being largely density-bounded. Figure 2 shows a general analytic relation corresponding to greater ISM density-bounding as the SFR increases.

On the other hand, while outflows are widely seen in starbursts (e.g., Martin 2003), searches for evidence of escaping ionizing radiation are puzzlingly low or absent (e.g., Leitherer et al. 1995; Heckman et al. 2001). Recently, direct detections of Lyman continuum emission on the order of a few percent have been reported in the blue compact dwarf galaxy Haro 11 (Bergvall et al. 2006), and a sample of Lyman break galaxies (Shapley et al. 2006). These observations are at odds with the indirect evidence presented here. One way to reconcile these results is by invoking a limited opening angle for the escape of this radiation (see comment by Pettini below).

The other possibility to explain the trend of lower WIM fraction with SFI, is that the ionizing sources are reduced, as mentioned above. We discuss this further in Oey et al. (2007), where we argue that a reduction in the field star population is unlikely. It may also be that greater extinction traps ionizing radiation near their sources. We have initiated an investigation using Spitzer and GALEX observations to evaluate this possibility.

\section{Summary}

In summary, we find that the softer, current generation of hot star atmospheres imply that our LMC HiI regions are largely radiation-bounded. If this result can be generalized to other star-forming galaxies, it will now likely require an additional source(s) to power the diffuse, warm ionized medium. On the other hand, our results from the SINGG survey suggest that the entire ISM of starburst galaxies are at least partly density-bounded, thereby allowing ionizing radiation to escape into their halos, and perhaps beyond, into the intergalactic medium. 


\section{Acknowledgements}

We gratefully acknowledge travel support from the IAU. This work was supported by NSF grant AST-0448893 and by NASA grant NAG5-10768.

\section{References}

Bergvall, N., Zackrisson, E., Andersson, B.-G., et al. 2006, A\&A A, 448, 513

Clarke, C. J. \& Oey, M. S. 2002, MNRAS, 337, 1299

Heckman, T. M., Sembach, K. R., Meurer, G. R., et al. 2001, ApJ, 558, 56

Hoopes, C. G., \& Walterbos, R. A.M. 2000, ApJ, 541, 597

Leitherer, C., Ferguson, H. C., Heckman, T. M., \& Lowenthal, J. 1995, ApJ, 454, L19

Martin, C. L. 2003, in: J. L. Rosenberg \& M. E. Putman (eds.), The IGM/Galaxy Connection:

The Distribution of Baryons at $z=0$, (Dordrecht: Kluwer), 205

Martins, F., Schaerer, D., \& Hillier, D. J. 2005, A\&SA, 436, 1049

Meurer, G. R., Hanish, D. J., Ferguson, H. C. et al. 2006, ApJS, 165, 307

Oey, M. S., \& Kennicutt, R. C., Jr. 1997, MNRAS, 291, 827

Oey, M. S., Meurer, G. R., Yelda, S. et al. 2007, ApJ, 661, 801

Panagia, N. 1973, AJ, 78, 929

Schaerer, D., \& de Koter, A. 1997, A\&A, 322, 598

Shapley, A. E., Steidel, C. C., Pettini, M., et al. 2006, ApJ, 651, 688

Smith, L. J., Norris, R. P.F., \& Crowther, P. A. 2002, MNRAS, 337, 1309

Thilker, D. A., Braun, R., \& Walterbos, R. A. M., 2000, AJ, 120, 2070

Vacca, W. D., Garmany, C. D., \& Shull, J. M. 1996, ApJ, 460, 914

Voges, E. S., Oey, M. S., Walterbos, R. A. M., \& Wilkinson, T. M. 2008, AJ, submitted

\section{Discussion}

GALlaGHER: Your results are consistent with our data for individual starbursts, that show little in the way of $\mathrm{H} \alpha$ emission beyond the starburst zone and associated wind. The mechanism, however, still isn't clear; preferential escape vs. dust blocking or something else. Do you have ideas for sharp tests?

OEY: We do have Spitzer and GALEX proposals in, to examine the SED's, including mid/far IR emission. Obvious tests to search for direct emission have been done by Leitherer and others, which are mostly negative.

LEITHERER: There is a rather straightforward test for probing the opacity of the diffuse ISM in these galaxies. It involves taking a UV longslit spectrum with STIS, which will hopefully be available in the next cycle. Since the Lyman continuum cannot be measured directly in these low- $z$ galaxies, take a heavy element like $\mathrm{C}$ or Si as a proxy. The STIS spectrum should give you a column density of the respective ion. With an assumption on ionization and abundance, you will get the opacity.

Pettini: A comment that the Shapley et al. (2006) results seem consistent with your suggestion that a narrow opening angle could be the way the radiation escapes. In their sample, most of the galaxies showed nothing, but in the two that were detected in the Lyman continuum, it looked like pretty much all of the radiation is escaping. Also, a second comment that the Bergvall results have been questioned recently by Grimes et al. (2007, ApJ 668, 891) 